\title{
Atividades gerenciais desempenhadas pelo enfermeiro no centro cirúrgico: obstáculos enfrentados pelo profissional no setor
}

\author{
Management activities performed by the nurse in the surgicenter: obstacles faced by the \\ professional in the sector
}

\begin{abstract}
Actividades gerenciales desempeñadas por el enfermero en el centro quirúrgico: obstáculos enfrentados por el profesional en el sector
\end{abstract}

\begin{abstract}
Maria de Jesus Monteiro da Silva ${ }^{1}$, Leonice dos Santos Nogueira ${ }^{2}$, Francisco Lucas de Lima Fontes $^{3^{*}}$, Ana Raquel Freitas dos Santos ${ }^{3}$, Janaina Ribeiro Corado ${ }^{3}$, Adryele Rianne Alves Lacerda ${ }^{3}$, Vanessa Maria Oliveira Viana ${ }^{4}$, Vera Alice Oliveira Viana ${ }^{1}$, Josélia Costa Soares ${ }^{3}$, Shirlane da Silva Barbosa $^{5}$, Emilene Paz Freitas ${ }^{1}$, Raifran Emanoel Silva Nunes ${ }^{6}$, Ariane Freire Oliveira ${ }^{7}$, Mércia Cycilia de França Lopes ${ }^{3}$, Walter Ney de Sousa Sales ${ }^{3}$.
\end{abstract}

\section{RESUMO}

Objetivo: Identificar as atividades gerenciais desempenhadas pelo enfermeiro no centro cirúrgico, bem como evidenciar também obstáculos enfrentados por esse profissional para execução de tais atividades no setor. Métodos: Trata-se de uma revisão bibliográfica narrativa descritiva, realizada por meio da Biblioteca Virtual em Saúde (BVS), Base de Dados de Enfermagem (BDEnf) e do buscador acadêmico Google Acadêmico, utilizando os descritores "centro cirúrgico", "administração hospitalar" e "enfermagem". Foram selecionados dez artigos para discussão, além de um Manual de Práticas Recomendadas. Resultados: O processo de gerenciamento envolve tanto o cuidado voltado à clientela quanto o manejo da unidade cirúrgica. A gerência do cuidado relaciona-se à busca pela qualidade assistencial e melhores condições de trabalho para os profissionais. O gerenciamento da unidade por sua vez engloba uma série de funções técnico-administrativas essenciais ao bom funcionamento do setor. Conclusão: Salienta-se a existência de obstáculos como relações conflituosas e falta de recursos materiais e humanos na rotina de trabalho do enfermeiro gerente de unidade cirúrgica, o que exige destes profissionais resiliência para superação dos desafios, capacidade de liderança e aprimoramento de sua gerência.

Palavras-chave: Centro cirúrgico, Administração hospitalar, Enfermagem.

\section{ABSTRACT}

Objective: To identify the managerial activities performed by the nurse in the surgical center, as well as to highlight obstacles faced by this professional to perform such activities in the sector. Methods: This is a descriptive narrative bibliographical review, performed through the Biblioteca Virtual em Saúde (BVS) database and the Base de Dados de Enfermagem (BDEnf) and the academic search engine Google Scholar, using the descriptors "centro cirúrgico", "gerenciamento" e "enfermagem". Ten articles for discussion were

${ }^{1}$ Centro Universitário Santo Agostinho. Teresina, Piauí, Brasil.

2 Unidade Integrada de Pós-graduação. Teresina, Piauí, Brasil.

${ }^{3}$ Faculdade UNINASSAU - Campus Redenção. Teresina, Piauí, Brasil. *E-mail:Iucasfontesenf@hotmail.com

${ }^{4}$ Universidade Federal do Piauí. Teresina, Piauí, Brasil.

${ }^{5}$ Associação de Ensino Superior do Piauí. Teresina, Piauí, Brasil.

${ }^{6}$ Faculdade FACID - Wyden. Teresina, Piauí, Brasil.

7 Centro Universitário UNINOVAFAPI. Teresina, Piauí, Brasil.

SUBMETIDO EM: 03/2019 
selected, as well as a Handbook of Best Practices. Results: The management process involves both customer care and surgical unit management. The management of care is related to the search for quality care and better working conditions for professionals. The management of the unit in turn encompasses a series of technical-administrative functions essential to the proper functioning of the sector. Conclusion: Obstacles such as conflictive relationships and lack of material and human resources in the work routine of the surgical unit manager nurse, which requires these professionals resilience to overcome the challenges, leadership capacity and improvement of their management.

Keywords: Surgicenter, Hospital administration, Nursing.

\section{RESUMEN}

Objetivo: Identificar las actividades gerenciales desempeñadas por el enfermero en el centro quirúrgico, así como evidenciar también obstáculos enfrentados por ese profesional para la ejecución de tales actividades en el sector. Métodos: Se trata de una revisión bibliográfica narrativa descriptiva, realizada por medio de las bases de datos Biblioteca Virtual em Saúde (BVS) y Base de Dados de Enfermagem (BDEnf) y del buscador académico Google Académico, utilizando los descriptores "centro cirúrgico", "administração hospitalar" e "enfermagem". Se seleccionaron diez artículos para discusión, además de un manual de prácticas recomendadas. Resultados: El proceso de gestión involucra tanto el cuidado orientado a la clientela como el manejo de la unidad quirúrgica. La gerencia del cuidado se relaciona con la búsqueda de la calidad asistencial y mejores condiciones de trabajo para los profesionales. La gestión de la unidad a su vez engloba una serie de funciones técnico-administrativas esenciales para el buen funcionamiento del sector. Conclusión: Se destaca la existencia de obstáculos como relaciones conflictivas y falta de recursos materiales y humanos en la rutina de trabajo del enfermero gerente de unidad quirúrgica, lo que exige de estos profesionales resiliencia para superar los desafíos, capacidad de liderazgo y perfeccionamiento de su gerencia.

Palabras-clave: Centro quirúrgico, Administración hospitalaria, Enfermería.

\section{INTRODUÇÃO}

A história do centro cirúrgico cruza-se com o desenvolvimento da Enfermagem perioperatória. Desde os primeiros procedimentos cirúrgicos, ocorridos devido a constantes guerras entre sociedades antigas, nas amputações de membros executadas pelos "cirurgiões barbeiros" a Enfermagem esteve presente, garantindo assistência aos envolvidos. Sua atuação englobava restrição e cuidados aos pacientes, além da viabilização de ambiente limpo. Com o descobrimento da anestesia, todo o processo cirúrgico evoluiu, tornando-se paulatinamente inovador e audacioso (SOBECC, 2013).

Hoje, o centro cirúrgico é um local complexo composto por profissionais de variadas especialidades onde são realizados os procedimentos anestésico-cirúrgicos. Por ser considerado um dos principais setores do hospital, requer elevado custo devido aos equipamentos de alta tecnologia e precisão, insumos e mão de obra qualificada. As atividades desenvolvidas no setor são tidas como de alto risco e criticidade, evidenciando necessidade de capacitação de seus profissionais para que não haja comprometimento da situação de saúde dos pacientes submetidos a cirurgias (DALCÓL C, GARANHANI ML, 2016).

O enfermeiro de centro cirúrgico tem sua prática reconhecida a cada dia pelos demais profissionais e pela sociedade. Atributos como liderança, conhecimento, organização, conduta ética e responsabilidade na prestação de uma assistência segura e isenta de danos foram essenciais para que o enfermeiro assumisse 0 controle das funções gerenciais deste setor (CAMPOS JAR et al., 2015).

A gerência dos serviços de saúde é uma prática exercida pelo enfermeiro há tempos, que adota os preceitos científicos e clássicos da Administração para gerir seu trabalho e os serviços sob sua supervisão (ROCHA T e ABRAHÃO AL, 2007). A atuação gerencial do enfermeiro nesses serviços deve considerar as 
dimensões assistencial, pedagógica, técnico-científica e política, assim como os elementos relacionados aos relacionamentos interpessoais, objetivando a garantia do que foi planejado para uma assistência holística e segura (SPAGNOL CA, 2005).

O enfermeiro, no papel de gerente, sempre enfrentará adversidades, o que exige deste profissional resiliência e planejamento para superação dos obstáculos advindos do processo de trabalho. Ressalta-se, portanto, a importância de identificação das funções gerenciais do enfermeiro no centro cirúrgico. Diante do explanado, o objetivo do presente estudo foi identificar as atividades gerenciais desempenhadas pelo enfermeiro no centro cirúrgico, bem como evidenciar também obstáculos enfrentados por esse profissional para execução de tais atividades no setor.

\section{MÉTODOS}

Estudo descritivo do tipo revisão de literatura bibliográfica narrativa, desenvolvida por meio da Biblioteca Virtual em Saúde (BVS), Banco de Dados de Enfermagem e do buscador virtual Google Acadêmico. Os descritores adotados para busca dos artigos foram: "centro cirúrgico", "administração hospitalar" e "enfermagem". Foram incluídos para construção desta revisão estudos disponíveis na íntegra, em língua portuguesa, publicados entre 2013 e 2017. Excluíram-se estudos incompletos, monografias, dissertações e teses. Após aplicação dos critérios, selecionaram-se dez estudos para edificação da discussão.

Ademais, também foi incorporado ao confronto literário o Manual de Práticas Recomendadas da Associação Brasileira de Enfermeiros de Centro Cirúrgico, Recuperação Anestésica e Centro de Material e Esterilização (SOBECC), por sua relevância ao tema explorado. Após a coleta dos estudos, partiu-se para uma leitura cuidadosa dos artigos selecionados, de modo a confirmar a sua relevância para a estruturação desta revisão; após essa fase trabalhou-se na leitura rigorosa dos fragmentos importantes à discussão; por fim, elaborou-se um quadro sinóptico com os dados retirados das fontes a fim de facilitar e organizar a discussão.

\section{RESULTADOS E DISCUSSÃO}

Conforme seleção dos artigos utilizados, segue a Tabela $1 \mathrm{com}$ distribuição de títulos dos estudos, autores/ano, tipos de estudo e principais conclusões.

O enfermeiro é o profissional capacitado a gerenciar todo o processo anestésico-cirúrgico e prestar assistência às necessidades do paciente durante o período perioperatório. A depender do porte do centro cirúrgico pode existir tanto o enfermeiro assistencial quanto o enfermeiro coordenador, que desempenha as funções gerenciais e técnico-administrativas (SOBECC, 2013).

$O$ processo de gerenciamento envolve tanto o cuidado voltado à clientela quanto o manejo da unidade cirúrgica. A gerência do cuidado relaciona-se à busca pela qualidade assistencial e melhores condições de trabalho para os profissionais, englobando a manutenção de ambiente cirúrgico seguro; recepção do paciente no centro cirúrgico; preenchimento de impressos próprios do setor; auxílio no posicionamento do paciente para o procedimento; realização de procedimentos como sondagem vesical; verificação da permeabilidade de cateteres, sondas e drenos; prestação de assistência ao paciente após o término da cirurgia e condução à sala de recuperação pós-anestésica (SANTOS JLG et al., 2013).

O gerenciamento da unidade, por sua vez, envolve o provimento de materiais, equipamentos e instrumentais cirúrgicos, além da orientação de manuseio e avaliação desses recursos; estabelecimento do perfil dos profissionais a serem admitidos; participação na seleção, integração e treinamento dos funcionários; controle da frequência dos profissionais, aferindo faltas, atrasos e licenças; elaboração das escalas de atividades dos funcionários; participação em reunião e comissões de integração com equipes multidisciplinares e tomada de decisões administrativas e assistenciais ancoradas em evidências científicas sobre o tema (SOUSA CS et al., 2013; SOBECC, 2013). 
Tabela 1 - Resultados dos artigos selecionados de acordo com título, autores/ano, tipo/abordagem de estudo e conclusões. Teresina, Piauí, Brasil, 2019.

\begin{tabular}{|c|c|c|c|}
\hline TÍTULO & AUTORES/ANO & TIPO DE ESTUDO & CONCLUSÃO \\
\hline $\begin{array}{l}\text { Práticas de enfermeiros na gerência } \\
\text { do cuidado em enfermagem e saúde: } \\
\text { revisão integrativa }\end{array}$ & $\begin{array}{l}\text { SANTOS JLG et } \\
\quad \text { al. (2013) }\end{array}$ & $\begin{array}{l}\text { Estudo qualitativo do } \\
\text { tipo revisão }\end{array}$ & $\begin{array}{l}\text { A gerência do cuidado realizada pelo enfermeiro relaciona-se diretamente à busca pela } \\
\text { qualidade assistencial e de melhores condições de trabalho, por meio de ações como: } \\
\text { realização do cuidado, gerência de recursos humanos e materiais, liderança, planejamento } \\
\text { da assistência, capacitação da equipe de enfermagem, coordenação da produção do } \\
\text { cuidado e avaliação das ações de enfermagem. }\end{array}$ \\
\hline $\begin{array}{l}\text { Avanços no papel do enfermeiro de } \\
\text { centro cirúrgico }\end{array}$ & $\begin{array}{l}\text { SOUSA CS et al. } \\
(2013)\end{array}$ & $\begin{array}{l}\text { Estudo qualitativo do } \\
\text { tipo revisão }\end{array}$ & $\begin{array}{l}\text { A reflexão deste tema incentiva o enfermeiro na busca da pesquisa, do desenvolvimento } \\
\text { técnico e da publicação sobre seus novos afazeres a fim de trazer por meio de parceria } \\
\text { entre os profissionais da prática e da academia avanços promissores na enfermagem } \\
\text { perioperatória. }\end{array}$ \\
\hline $\begin{array}{l}\text { Conhecendo as formas de cuidar dos } \\
\text { enfermeiros de centro cirúrgico - uma } \\
\text { construção a partir da teoria } \\
\text { fundamentada nos dados }\end{array}$ & $\begin{array}{l}\text { SANTOS FK et al. } \\
(2014)\end{array}$ & Estudo qualitativo & $\begin{array}{l}\text { Concluiu-se que os enfermeiros de centro cirúrgico integram diversos fatores, atuando, por } \\
\text { vezes, como agentes do cuidado indireto, apesar das dificuldades de um contexto complexo } \\
\text { e específico. }\end{array}$ \\
\hline $\begin{array}{l}\text { Cultura de segurança entre } \\
\text { profissionais de centro cirúrgico }\end{array}$ & $\begin{array}{l}\text { CAUDURO FLF et } \\
\text { al. (2015) }\end{array}$ & $\begin{array}{l}\text { Estudo quantitativo do } \\
\text { tipo survey }\end{array}$ & $\begin{array}{l}\text { Os resultados apontam a necessidade de ações com vistas a fortalecer a cultura de } \\
\text { segurança do paciente considerando a complexidade da assistência prestada em centro } \\
\text { cirúrgico. }\end{array}$ \\
\hline $\begin{array}{l}\text { Percepção da equipe de enfermagem } \\
\text { do centro cirúrgico acerca da } \\
\text { acreditação hospitalar em um hospital } \\
\text { universitário }\end{array}$ & $\begin{array}{l}\text { FERNANDES } \\
\text { HMLG e PENICHE } \\
\text { ACG (2015) }\end{array}$ & Estudo quantitativo & $\begin{array}{l}\text { Este diagnóstico situacional subsidiará a reestruturação dos pontos vulneráveis avaliados } \\
\text { nas três dimensões, sobretudo os da dimensão de estrutura, com vistas à acreditação nível } \\
2 \text { pela Organização Nacional de Acreditação pleiteada na Instituição. }\end{array}$ \\
\hline $\begin{array}{l}\text { Exercício da liderança do enfermeiro } \\
\text { no bloco cirúrgico }\end{array}$ & $\begin{array}{l}\text { BRAGA DD et al. } \\
(2016)\end{array}$ & Estudo qualitativo & $\begin{array}{l}\text { O exercício da liderança destes profissionais está atrelado ao diálogo, mas ainda são } \\
\text { necessários movimentos que auxiliem na superação das dificuldades e que potencializem } \\
\text { esta competência profissional no ambiente de trabalho. }\end{array}$ \\
\hline $\begin{array}{l}\text { Representação da prática gerencial } \\
\text { do enfermeiro na unidade de } \\
\text { internação: perspectiva da equipe de } \\
\text { enfermagem }\end{array}$ & $\begin{array}{l}\text { LIMA RS et al. } \\
\qquad(2016)\end{array}$ & Estudo qualitativo & $\begin{array}{l}\text { A gerência, apreendida como processo aparte do cuidado, é qualificada como prática não } \\
\text { familiar, de difícil compreensão e valoração. }\end{array}$ \\
\hline $\begin{array}{l}\text { Centro cirúrgico: desafios e } \\
\text { estratégias do enfermeiro nas } \\
\text { atividades gerenciais }\end{array}$ & $\begin{array}{l}\text { MARTINS FB e } \\
\text { DALL'AGNOLB } \\
\text { CM et al. (2016) }\end{array}$ & Estudo quantitativo & $\begin{array}{l}\text { Ponderou-se que as atividades gerenciais envolvem a promoção de momentos dialógicos } \\
\text { para articular os diferentes processos existentes no CC, a fim de produzir subsídios para } \\
\text { ampliar a segurança e a qualidade nos serviços prestados. }\end{array}$ \\
\hline $\begin{array}{l}\text { Conflitos e dilemas éticos: vivências } \\
\text { de enfermeiras no centro cirúrgico }\end{array}$ & $\begin{array}{l}\text { OLIVEIRA MAN e } \\
\text { ROSA DOS (2016) }\end{array}$ & $\begin{array}{l}\text { Estudo qualitativo do } \\
\text { fenomenológico }\end{array}$ & $\begin{array}{l}\text { Embora com dificuldade para reconhecer os conflitos e dilemas éticos, tomam decisões e, } \\
\text { ao fazê-lo, procuram manter o equilíbrio emocional da equipe e a harmonia no ambiente de } \\
\text { trabalho. }\end{array}$ \\
\hline $\begin{array}{l}\text { Relações interpessoais no centro } \\
\text { cirúrgico sob a ótica da enfermagem: } \\
\text { estudo exploratório }\end{array}$ & $\begin{array}{c}\text { TRAJANO CMF et } \\
\text { al. (2017) }\end{array}$ & Estudo qualitativo & $\begin{array}{l}\text { Contemplar as relações interpessoais, entre os profissionais no centro cirúrgico, contribuir } \\
\text { para instrumentalizar a gestão, para estabelecer estratégias de enfrentamento e para } \\
\text { assegurar um trabalho em equipe, em ambiente harmônico, que preste uma assistência } \\
\text { integral, humanizada e de segurança ao paciente em situação crítica. }\end{array}$ \\
\hline
\end{tabular}

Fonte: próprios autores, 2019.

REAS/EJCH | Vol. Sup. 17 e652 | DOI: https://doi.org/10.25248/reas.e652.2019 Página 4 de 6 
O gerenciamento de Enfermagem precede a tomada de decisões, que difere de acordo com o grau de autonomia do enfermeiro gerente e de como ocorre sua relação com a equipe e com a política institucional, repercutindo na forma de desenvolver o seu processo de trabalho (MARTINS FZ e DALL'AGNOL CM, 2016). A tomada de decisão é fundamental para o gerenciamento de Enfermagem na unidade cirúrgica. Para realizar suas atividades, o enfermeiro gerente de centro cirúrgico necessita manter-se atualizado e diariamente avaliar suas ações de acordo com a missão, a visão e os valores institucionais. A gerência, apreendida como processo à parte do cuidado, é qualificada como prática estranha e não corriqueira, de difícil entendimento e pouco reconhecimento (LIMA RS et al., 2016).

Além da execução de atividades já elencadas, o enfermeiro também enfrenta desafios na gerência do centro cirúrgico. O estudo de Fernandes HMLG e Peniche ACG (2015) analisou a percepção da equipe de Enfermagem acerca do processo de acreditação hospitalar no centro cirúrgico em um hospital universitário e expôs que o maior entrave na gerência do centro cirúrgico seria a instalação de uma cultura de segurança, pois esta exigia envolvimento integral de todos os profissionais atuantes no setor, desde a assistência ao apoio.

Um estudo que avaliou a percepção da cultura de segurança entre profissionais de saúde atuantes em unidades de centro cirúrgico evidenciou que os profissionais percebiam nas gerências da unidade cirúrgica e do hospital lacunas quanto à promoção da cultura de segurança do paciente, fato que representa um empecilho à organização (CAUDURO FLF et al., 2015). Intervenções sólidas como garantia de condições adequadas de trabalho, educação continuada e estímulo ao comprometimento profissional podem ser estratégias úteis na cultura de segurança dentro centro cirúrgico. As condições de trabalho da instituição, como materiais e insumos, também são capazes de influenciar a dinâmica de trabalho no centro cirúrgico, considerando que a assistência possui uma dimensão técnica. Faz-se necessário avaliar a real situação ao desempenhar qualquer tipo de trabalho junto aos pacientes diante das limitações próprias à instituição (SANTOS FK et al., 2014).

Segundo Martins FZ e Dall'Agnol CM (2016), os desafios e limitações encontrados nas atividades gerenciais no centro cirúrgico derivam de condições intrínsecas do próprio ambiente, marcada pela imprevisibilidade e pela necessidade constante de planejamento e organização das ações. Aliada a essas condições estão a falta de material e equipamentos adequados, falta de recursos humanos, os conflitos existentes entre as equipes onde o enfermeiro deverá ter o poder de apaziguar, além das limitações da equipe médica na compreensão do trabalho da Enfermagem.

De acordo com Trajano MFC et al. (2017) as relações interpessoais podem ser afetadas por comunicação prejudicada, presentes em modelos de gestão hierarquizada; sobrecarga de trabalho e exigência de prontidão às atribuições. Esses aspectos contribuem para relações conflituosas. A presença de conflitos no centro cirúrgico ocorre devido a existência de grupos singulares com linhas de pensamento diferentes do mesmo mundo. O enfermeiro ao gerenciar conflitos deve utilizar-se do seu poder de liderança, diálogo, negociação e trabalho em equipe. Nesse caminho é relevante que os profissionais relacionem-se de modo a alcançar seus objetivos (OLIVEIRA MAN e ROSA DOS, 2016).

O estudo de Braga DD et al. (2016) que objetivou conhecer a percepção de enfermeiros atuantes em bloco cirúrgico sobre o exercício da liderança nesse setor, mostrou que os enfermeiros seguem uma liderança pautada no diálogo, ancorada pela confiança entre os profissionais. Este estudo destacou ainda que, entre as dificuldades que atingem o exercício da liderança, encontram-se a formação acadêmica e as relações interpessoais prejudicadas. Estratégias como uso da autonomia, suporte da chefia e educação continuada facilitam a execução da liderança do enfermeiro.

\section{COSIDERAÇÕES FINAIS}

Por meio dessa revisão fica evidente que a produção científica sobre o tema não acompanha o ganho de espaço profissional que o enfermeiro gerente conquista. Esse estudo teve como limitação as poucas produções científicas encontradas que tratavam da gerência de Enfermagem no centro cirúrgico, o que 
dificultou, de fato, sua discussão. Infere-se a necessidade de maiores estudos sobre o assunto a fim de obtenção de uma realidade sobre a atuação gerencial do enfermeiro no centro cirúrgico. Mesmo com ampla presença e difusão de conteúdo em livros, artigos sobre o tema são escassos, o que justifica a utilização na discussão do Manual de Práticas Recomendadas da Associação Brasileira de Enfermeiros de Centro Cirúrgico, Recuperação Anestésica e Centro de Material e Esterilização, essencial para as boas práticas na rotina do centro cirúrgico. Ademais, salienta-se a existência de obstáculos como relações conflituosas e falta de recursos materiais e humanos na rotina de trabalho do enfermeiro gerente de unidade cirúrgica, o que exige destes profissionais resiliência para superação dos desafios, capacidade de liderança e aprimoramento de sua gerência.

\section{REFERÊNCIAS}

1. ASSOCIAÇÃO BRASILEIRA DE ENFERMEIROS DE CENTRO CIRÚRGICO, RECUPERAÇÃO ANESTÉSICA E CENTRO DE MATERIAL E ESTERILIZAÇÃO (SOBECC). Práticas recomendadas SOBECC. 6a ed. São Paulo: Manole, 2013.

2. BRAGA DD et al. Exercício da liderança do enfermeiro no bloco cirúrgico. Journal of Nursing and Health, 2016; 6(2): 267-278.

3. CAMPOS JAR et al. Produção científica da enfermagem de centro cirúrgico de 2003 a 2013. Revista SOBECC, 2015; 20(2): 81-95.

4. CAUDURO FLF et al. Cultura de segurança entre profissionais de centro cirúrgico. Cogitare Enfermagem, 2015; 20(1): 129-138.

5. DALCÓL C, GARANHANI ML. Papel gerencial do enfermeiro de centro cirúrgico: percepções por meio de imagens. Revista Eletrônica Enfermagem, 2016; 34:e1118.

6. FERNANDES HMLG, PENICHE ACG. Percepção da equipe de enfermagem do Centro Cirúrgico acerca da Acreditação Hospitalar em um Hospital Universitário. Revista Escola de Enfermagem da USP, 2015; 49(Esp): 22 -28.

7. GIL AC. Capítulo 5: Como delinear uma pesquisa bibliográfica. In: Como elaborar projetos de pesquisa. 6ª ed. São Paulo: Atlas, 2017.

8. LIMA RS et al. Representação da prática gerencial do enfermeiro na unidade de internação: perspectiva da equipe de enfermagem. Revista Gaúcha de Enfermagem, 2016; 37(1): e54422.

9. MARCONI MA, LAKATOS EM. Técnicas de pesquisa: planejamento e execução de pesquisas, amostragens e técnicas de pesquisas, elaboração, análise e interpretação de dados. 8aㅡ ed. São Paulo: Atlas, 2017.

10. MARTINS FZ, DALL'AGNOL CM. Centro cirúrgico: desafios e estratégias do enfermeiro nas atividades gerenciais. Revista Gaúcha de Enfermagem, 2016; 37(4): e56945.

11. OLIVEIRA MAN, ROSA DOS. Conflitos e dilemas éticos: vivências de enfermeiras no centro cirúrgico. Revista Baiana de Enfermagem, 2016; 30(1): 344-355.

12. ROCHA T, ABRAHÃO AL. A inserção das tecnologias leves, leves duras e duras no gerenciamento do cuidado pelos enfermeiros de um hospital universitário. Online Brazilian Journal of Nursing, 2007; 6(2).

13. SANTOS FK et al. Conhecendo as formas de cuidar dos enfermeiros de centro cirúrgico - uma construção a partir da teoria fundamentada nos dados. Texto \& Contexto Enfermagem, 2014; 23(3): 696-703.

14. SANTOS JLG et al. Práticas de enfermeiros na gerência do cuidado em enfermagem e saúde: revisão integrativa. Revista Brasileira de Enfermagem, 2013; 66(2): 257-263.

15. SOUSA CS et al. Avanços no papel do enfermeiro de centro cirúrgico. Revista de Enfermagem da UFPE, 2013; 7(Esp): 6288-6293.

16. SPAGNOL CA. (Re)pensando a gerência em enfermagem a partir de conceitos utilizados no campo da Saúde Coletiva. Ciência \& Saúde Coletiva, 2005; 10(1): 119-127.

17. TRAJANO MFC et al. Relações interpessoais no centro cirúrgico sob a ótica da enfermagem: estudo exploratório. Online Brazilian Journal of Nursing, 2017; 16(1): 159-169. 